\title{
A Hybrid MI-SSVEP based Brain Computer Interface for Potential Upper Limb Neurorehabilitation: A Pilot Study
}

McGeady, Ciaran; Vuckovic, Aleksandra; Puthusserypady, Sadasivan

Published in:

Proceedings of 2019 7th International Winter Conference on Brain-Computer Interface

Link to article, DOI:

10.1109/IWW-BCI.2019.8737333

Publication date:

2019

Document Version

Peer reviewed version

Link back to DTU Orbit

Citation (APA):

McGeady, C., Vuckovic, A., \& Puthusserypady, S. (2019). A Hybrid MI-SSVEP based Brain Computer Interface for Potential Upper Limb Neurorehabilitation: A Pilot Study. In Proceedings of 2019 7th International Winter Conference on Brain-Computer Interface IEEE. https://doi.org/10.1109/IWW-BCI.2019.8737333

\section{General rights}

Copyright and moral rights for the publications made accessible in the public portal are retained by the authors and/or other copyright owners and it is a condition of accessing publications that users recognise and abide by the legal requirements associated with these rights.

- Users may download and print one copy of any publication from the public portal for the purpose of private study or research.

- You may not further distribute the material or use it for any profit-making activity or commercial gain

- You may freely distribute the URL identifying the publication in the public portal 


\section{A Hybrid MI-SSVEP based Brain Computer Interface for Potential Upper Limb Neurorehabilitation: A Pilot Study}

\author{
Ciarán McGeady \\ Division of Biomedical Engineering \\ University of Glasgow \\ Glasgow, United Kingdom \\ c.mcgeady.1@ research.gla.ac.uk
}

\author{
Aleksandra Vučković \\ Division of Biomedical Engineering \\ University of Glasgow \\ Glasgow, United Kingdom \\ aleksandra.vuckovic@glasgow.ac.uk
}

\author{
Sadasivan Puthusserypady \\ Department of Electrical Engineering \\ Technical University of Denmark (DTU) \\ Lyngby, Denmark \\ spu@elektro.dtu.dk
}

\begin{abstract}
This pilot study implements a hybrid BCI system in an effort to deduce the effects of measuring more than one brain signal in a motor imagery (MI) task. In addition to sensorimotor rhythms (SMRs), a steady state visual evoked potential (SSVEP) was introduced to acquire additional information relating to user intention. A common spatial pattern (CSP) filter followed by a support vector machine (SVM) classifier were used to distinguish between $\mathrm{MI}$ and the resting state. The power spectral density (PSD) was used to classify the SSVEP. Results from online simulations of EEG data collected from 10 able-bodied participants showed that the hybrid BCI's performance achieved a classification accuracy of $77.3 \pm 8.2 \%$, with an SSVEP classification accuracy of $94.4 \pm 3.5 \%$, and MI classification accuracy of $80.9 \pm 8.1 \%$, an improvement upon purely MI-based multi-class BCI paradigms.
\end{abstract}

Index Terms-Brain Computer Interface (BCI), Electroencephalogram (EEG), Steady State Visually Evoked Potential (SSVEP), Common Spatial Patterns (CSP), Motor Imagery (MI), Hybrid, Neurorehabilitation.

\section{INTRODUCTION}

Stroke affects around 17 million people every year, making it one of the world's major sources of acquired disability [1]. A stroke occurs when there is a sudden interruption to the flow of oxygen-rich blood to a region of the brain. Without a supply of oxygen, brain tissues begin to die after only a few minutes, leading to persistent functional deficits, such as the motor impairment of the upper and lower limbs [2]. Despite lasting hemiparesis, patients have been found to make dramatic motor recoveries due to brain neuroplasticity. This phenomenon underpins the majority of repetitive, goal-based rehabilitative practice as mimicking natural motor patterns may result in the rewiring and strengthening of the motor neural networks resulting in the restoration of normal motor function [3]. However, since some stroke survivors have no residual limb movement in their affected side, they are unable to physically execute tasks. Motor imagery (MI), which involves the imagination of movements, offers an alternative, as many of the same neural regions are involved in MI as in actual movement [4]. Unlike physical practice, however, it is impossible for a clinician to properly gauge a patient's compliance to MI tasks. Brain-computer interface (BCI) technology can remedy this by interpreting brain signals relating to MI from electroencephalogram (EEG) data. Bandpower within the $\mu(8-12 \mathrm{~Hz})$ and $\beta(13-30 \mathrm{~Hz})$ bands have been shown to desynchronize during movement and motor imagery, allowing for the classification of EEG signals relating to different MI states [5]. BCI can offer performance-dependent feedback, providing clinicians with quantitative data, whilst encouraging active participation from patients, an important feature in improving patient adherence and outcomes [6].

Numerous pilot studies exploring the feasibility of using BCI for upper limb rehabilitation after stroke have been coupled with other interventions, like orthotic feedback or functional electrical stimulation (FES) [7]-[11]. Providing contingent sensory feedback reestablishes the sensorimotor loop as the feedback is dependent on the detection of correct brain activity, leading to neuroplasticity and motor recovery. Notable clinical work includes a randomised controlled trial by Ramos-Murguialday et al. who used BCI to detect MI in 30 chronic stroke patients [12]. Whilst engaged in the training, half of the population received genuine orthotic feedback based on their performance whilst the other half received random feedback. Both groups also received standard physiotherapy. Results showed significant improvements in volitional movement in the former group, but none in the latter, likely owing to the lack of contingent feedback in the latter group [12]. More recently, Biasiucci et al. reported even larger motor improvements with their controlled trial using BCI-FES therapy on 27 chronic moderate-severe stroke patients [13]. Patients were given either BCI-FES or sham-FES therapy. Only the former group benefited from the intervention, with some patients seeing motor recovery 15 years after their initial stroke, and, most interestingly of all, benefits remained 6-12 months after the therapy. Although a BCI approach to neurorehabilitation is relatively novel, preliminary results illustrate how a BCI, when combined with contingent activation of afferent pathways, can drive purposeful plasticity resulting in significant functional recovery.

The BCI paradigms used in the majority of clinical trials 
have involved a two class approach, i.e. the system would classify brain signatures relating to two mental states, usually MI and the resting state [12], [13]. Ideally, BCI systems would have more classes, increasing the degree of control over a given application. However, from a signal processing perspective, the ability to resolve classes becomes increasingly difficult with each additional class, especially when the classes relate to movements produced by regions of the motor cortex which are close to one another, in the case of multiple gestures of the same hand, for instance [14]. Indeed, a study by Palma et al. aimed to solve a 3-class MI problem concerning gestures of the right upper limb in able-bodied participants: namely, palmar grasp, pinch, and elbow flexion [15]. Although they achieved classification accuracies of nearly $65 \%$, this would not be practical in a rehabilitation setting, falling below the commonly held $70 \%$ threshold for BCI efficiency [16]. Any lower than this would result in great user frustration [17].

To maintain good performance while increasing the degrees of freedom a user has over a BCI system, this study explored hybrid BCI technology. A hybrid system interprets more than one brain signal, allowing clinicians to infer more information relating to user intentions per unit of time [18]. Hybrid BCI's can be implemented in a variety of ways: they may classify two signals sequentially; where the first activates the system, and the second allows selection of an output [19]; or simultaneously, which requires the user to perform multiple tasks at the same time [20]. This pilot study investigates the feasibility of combining a 2-class MI paradigm with another mental task. We hypothesised that the inclusion of a visual attention task in a MI-based BCI paradigm would come at no significant cost to the user in terms of mental demand, allowing them to provide the system with more information relating to their intentions. We chose steady state visually evoked potentials (SSVEPs) - a time-locked region of positive or negative deflection amongst EEG data at around the same frequency of a periodic stimuli [21] - as our additional signal as it is highly robust and reliable, often providing accuracies well in excess of $90 \%$ [22].

\section{Materials And Methods}

\section{A. Data collection and experimental paradigm}

Ten able-bodied volunteers ( $32 \pm 15$ years old, 5 males, 5 females) took part in an initial data collection session to calibrate their individual spatial filter and classifier for differentiating between two mental states: right hand MI and resting. EEG was recorded at a sampling frequency of 256 $\mathrm{Hz}$ with a total of 16 active surface electrodes. To capture activity relating to $\mathrm{MI}, 10$ electrodes were placed above the sensorimotor cortex at C1, C2, C3, C4, C5, C6, FC3, FC4, $\mathrm{CP} 3$, and CP4, according to the international 10-20 system. To capture activity relating to SSVEPs, 6 electrodes were placed over the parietal-occipital region at $\mathrm{O} 1, \mathrm{Oz}, \mathrm{O} 2, \mathrm{PO} 7, \mathrm{PO}$, and Pz. Only two of these six electrodes were used to create a bipolar channel for SSVEP detection; Oz-Pz was the default, however, due to subject variability, occasionally this channel was inadequate, and the other channels were explored. The ground electrode was placed at Fpz and the reference electrode was placed on the left earlobe. The amplifier used was the g.USBamp by g.tec and was set to apply a band-pass filter from 0.5 to $100 \mathrm{~Hz}$, with a notch filter at $50 \mathrm{~Hz}$ to reduce power line noise.

All subjects provided written informed consent prior to their participation in the experiments, which were approved by the Regional Committee on Health Research Ethics for the Capital Region of Denmark (reference H-3-2013-004) and carried out in accordance with the corresponding guidelines and relevant regulations on the use of human subjects for health-related scientific research.

Only the experimental supervisor and volunteer were present in a sound-proof room during the experimental sessions. Inside the room, the lights were dimmed and the test subject was seated $60 \mathrm{~cm}$ away from a computer screen presenting visual cues and stimuli. During each trail the participants were required to mirror the gestures shown on the screen; first, in a movement execution (ME) session (where they physically performed the gesture), then an MI session (where they imagined performing the gesture). In a session there were 45 trials of $6 \mathrm{~s}$. A $4 \mathrm{~s}$ period of rest and refocus followed each trial. Figure 1 shows the timing scheme for each trail. To ensure focus, each trial began with participants gazing at a fixation cross at the center of the screen for $2 \mathrm{~s}$; this was accompanied by a $1 \mathrm{~s}$ tone. Then, randomly, one of the visual cues would appear for $6 \mathrm{~s}$, during which time the participants were instructed to hold the gesture for as long it was present on screen. Figure 2 shows the goal-based visual cues given during each trial: palmar grasp, pinch and point. The use of these gestures was motivated by the fact that hand movements like these are critical for daily life and so would be useful in a rehabilitation setting. To limit participant fatigue, the calibration sessions were split into 5 sub-sessions of 18 trials each, with a 2 minute breaks between each sub-session.

A hybrid session followed where each participant was instructed to follow the same routine as before with the added task of simultaneously focusing their gaze on one of three flickering light-emitting diodes (LEDs), each strategically positioned around the computer screen. Figure 2 shows each goal-based cue that was presented on screen, with an arrow showing the direction of the corresponding visual stimuli. The timing of the hybrid trials was the same as the calibration session: $6 \mathrm{~s}$ per trial with a $4 \mathrm{~s}$ inter-trial interval. Again, there were 5 sub-sessions of 18 trials.

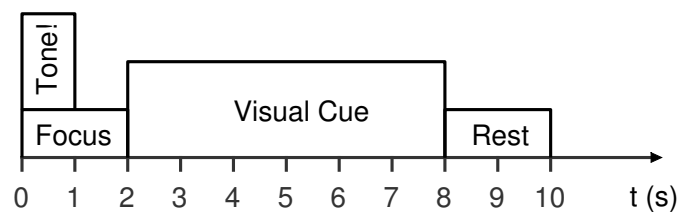

Fig. 1. Timing of a trial. At $0 \mathrm{~s}$, a fixation cross appeared on screen for $2 \mathrm{~s}$ and a tone played for $1 \mathrm{~s}$. After these $2 \mathrm{~s}$ a visual cue was randomly presented for $6 \mathrm{~s}$, which was followed by a $2 \mathrm{~s}$ rest period. Afterwards the sequence repeated for the remaining number of trials. 


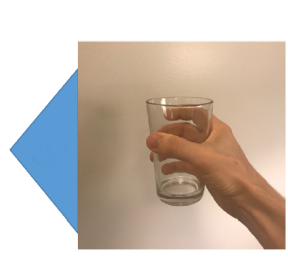

(a) Palmar grasp

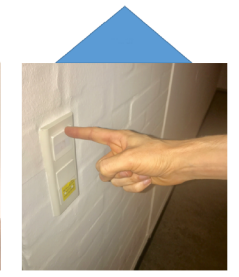

(b) Point

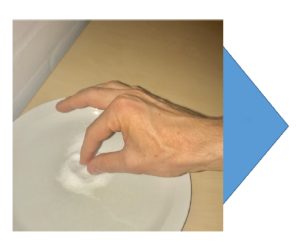

(c) Pinch

Fig. 2. Goal-based visual cues given during each trial. An arrow accompanies each gesture to indicate which of the three SSVEP targets to focus on. Arrows only apply during the hybrid sessions.

\section{B. Pre-processing}

'EEGlab' was used offline to split the calibration data into epochs relating to each trial. The data was cleaned by visual inspection and noisy trials were removed manually. Around 5 epochs were removed for each subject. For each participant, their calibration data was used to build a common spatial pattern (CSP) filter. The CSP technique is based on the simultaneous diagonalization of two covariance matrices. It spatially filters new EEG data, creating a new time series whose variances are optimal for the separation of EEG data relating to two mental states. The mathematical procedure we used to calculate the CSP filter has been previously described [23]. We used 4 pairs of filters for feature extraction, as this was determined to be optimal through experimentation. The log-variances of the spatially filtered EEG signals were used as input features to create a support vector machine (SVM), given the efficiency that this classifier has shown in previous work.

\section{Hybrid classification}

The hybrid session data was used offline to simulate an online system. For each subject, a sliding window of $2 \mathrm{~s}$ (512 sampling points) moved in $1 \mathrm{~s}$ intervals across the hybrid EEG data, simulating new incoming data. EEG classification was performed with each shift of the window. The SSVEPs were classified by using a fast Fourier transform (FFT) to estimate the power spectrum density around the three LED frequencies $(13,15,18 \mathrm{~Hz})$ and their first harmonics $(26,30,36 \mathrm{~Hz})$. The harmonic sums were computed and the most powerful frequency was presumed to be the intended class. If the class assigned to the window matched the actual trial frequency, then the system would proceed to the classification of the sensorimotor rhythm (SMR) component of the signal. This involved filtering with a band-pass filter across the $\mu$-band (8$12 \mathrm{~Hz}$ ) (which was found to be the most reactive frequency band), and the individually-calculated CSP filter. The logvariance of the spatially filtered EEG signal was used as a feature to classify the window with the SVM. A successfully classified window was defined as the correct classification of each component of the hybrid condition simultaneously. Therefore, system accuracy was defined as the number of correctly classified windows per session.

TABLE I

ONLINE CLASSIFICATION ACCURACIES (\%) FOR THE MOTOR EXECUTION AND MOTOR IMAGERY TASKS.

\begin{tabular}{|c|c|c|c|c|c|c|}
\hline \multirow{2}{*}{ Subject } & \multicolumn{3}{|c|}{ Motor Execution } & \multicolumn{3}{c|}{ Motor Imagery } \\
\cline { 2 - 7 } & SMR & SSVEP & Hybrid & SMR & SSVEP & Hybrid \\
\hline 1 & 72.5 & 88.9 & 70.0 & 78.9 & 90.6 & 75.3 \\
2 & 75.0 & 98.1 & 73.0 & 77.9 & 100.0 & 77.9 \\
3 & 74.5 & 93.7 & 74.1 & 85.4 & 94.1 & 79.1 \\
4 & 80.0 & 95.7 & 77.6 & 88.2 & 95.1 & 82.3 \\
5 & 88.2 & 94.1 & 82.3 & 86.7 & 93.7 & 83.2 \\
6 & 86.3 & 92.9 & 84.3 & 85.9 & 94.1 & 83.0 \\
7 & 76.0 & 96.0 & 72.7 & 64.3 & 95.1 & 60.8 \\
8 & 84.7 & 94.6 & 79.2 & 84.3 & 93.7 & 78.0 \\
9 & 78.4 & 99.6 & 76.5 & 87.5 & 100.0 & 87.5 \\
10 & 66.2 & 89.0 & 58.6 & 70.1 & 89.0 & 66.3 \\
\hline \hline Mean & 77.2 & 94.3 & 74.8 & 80.9 & 94.4 & 77.3 \\
Std & 6.8 & 3.6 & 7.2 & 8.1 & 3.5 & 8.2 \\
\hline
\end{tabular}

\section{RESULTS}

The classification accuracies of the online simulation for all 10 participants are reported in Table I. Given also are the accuracies of the constituent signals if they were classified individually. The hybrid BCI performed poorer than the two component signals. This was expected as only a simultaneous correct classification of the two component signals counted as a successful hybrid classification. The average hybrid accuracy of the MI-SSVEP sessions was $77.3 \pm 8.2 \%$, and $74.8 \pm 7.2 \%$ for the ME-SSVEP sessions. A paired t-test analysis showed that the difference between these results are not statistically significant $(\mathrm{P}>0.05)$.

SSVEP classification was highly accurate and consistent among participants, with a mean accuracy of $94.4 \pm 3.4 \%$ and $94.3 \pm 3.6 \%$ for the MI-SSVEP and ME-SSVEP sessions, respectively $(\mathrm{P}>0.05)$. No subjects were deemed SSVEP illiterate; Subject 2 and 9 even had 100\% SSVEP classification accuracies for their MI-hybrid run. This was an expected and welcome result as it allowed MI classification to mostly dictate the hybrid accuracy whilst increasing the degrees of freedom available to the BCI system.

There was much subject variability amongst the MI-hybrid results. Subject 9 returned excellent results, with an accuracy of $87.5 \%$. Whereas, Subject 7 and 10 would be considered 'BCI illiterate', as both achieved results below the illiteracy threshold (70\%), with accuracies of $60.8 \%$ and $66.3 \%$, respectively.

All subjects performed with ME-hybrid accuracies above the commonly accepted threshold for BCI illiteracy, except for Subject 1, whose performance was exactly on the boundary between literacy and illiteracy; and, again, Subject 10, whose poor result of $58.6 \%$ would make them unsuitable for BCI training.

During the hybrid sessions the participants were instructed to perform both the MI (or ME) and SSVEP task simultaneously for the duration of each $6 \mathrm{~s}$ trial. The accuracy with respect to the length of a trail is illustrated in Figure 3 and 4. The SSVEP condition can be seen to be consistently providing high accuracies $(>95 \%)$ for the duration of the trial. The 


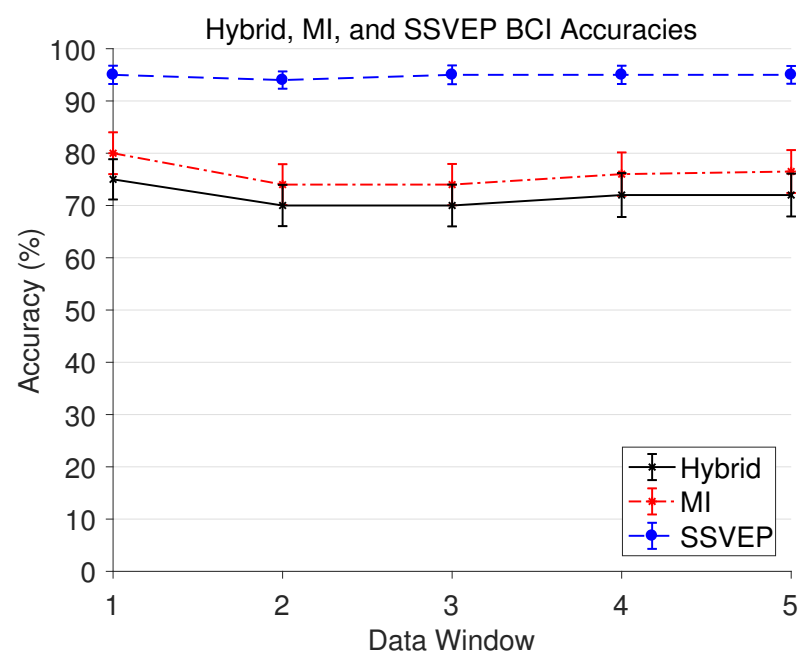

Fig. 3. Average accuracy across all trails and participants. Each data point represents the accuracy of one of the five $2 \mathrm{~s}$ data windows classified during each MI-SSVEP trial. The first point represents a two second window from the onset of the cue at $t=0 s$ to $t=2 s$, the second point spans from $t=1 s$ to $\mathrm{t}=3 \mathrm{~s}$, and so on.

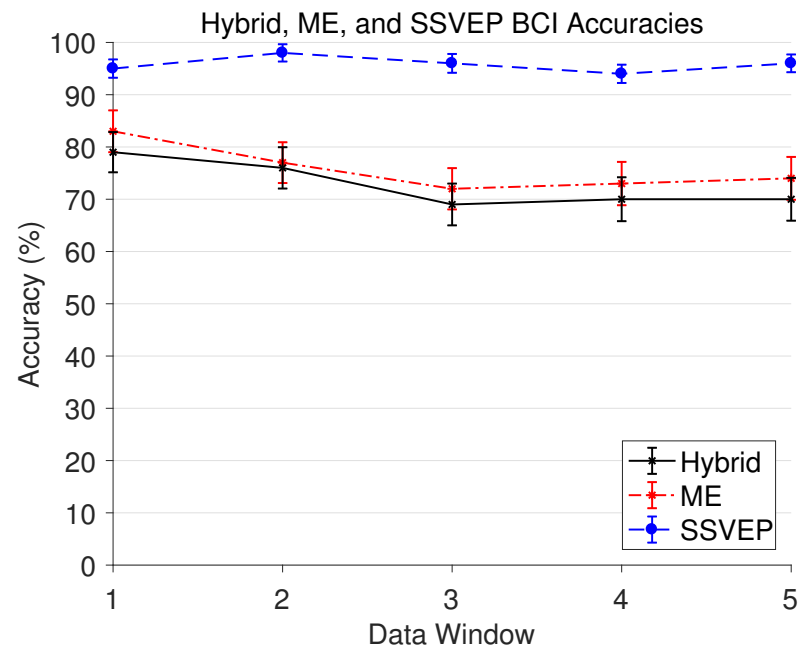

Fig. 4. Average accuracy across all trails and participants. Each data point represents the accuracy of one of the five $2 \mathrm{~s}$ data windows classified during each ME-SSVEP trial. The first point represents a two second window from the onset of the cue at $t=0 s$ to $t=2 s$, the second point spans from $t=1 s$ to $\mathrm{t}=3 \mathrm{~s}$, and so on.

hybrid and SMR components are less stable, varying between $68 \%$ and $84 \%$, and appear to decrease in accuracy with time, but not significantly so.

\section{DISCUSSION}

The accuracy of the MI component from the hybrid paradigm was $80 \pm 8.1 \%$, consistent with the literature reporting on CSP algorithms for 2-class MI problems. In fact, this was better than the $75.5 \pm 18.2 \%$ mean accuracy reported by Lotte et al. in their classical CSP approach to classification of three BCI competition data sets [24]. This affirms the work of Brunner et al. [20]: simultaneous classification of a secondary brain signal does not necessarily have a negative impact on MI accuracy. Also consistent was the high subject variability, including poor performances by some subjects. Subject 7 and 10 did not produce SMR modulations suitable for an online system, with accuracies less than the threshold for BCI illegibility. This is in line with the often-reported proportion of illiterate participants in group studies: 20\% [16]. Interestingly, the MI hybrid outperformed the ME hybrid. This is a surprising result given that most participants mentioned the added effort required of MI over ME. Perhaps more artifacts were introduced during ME session due to the explicit motion, although the $40 \mathrm{~Hz}$ upper cutoff frequency of the bandpass filter should have removed muscle artifacts.

One of the objectives of this project was to deduce whether a hybrid BCI could provide a more accurate system than purely MI-based multi-class BCIs. Palma et al. designed a similar paradigm to the one in this project, classifying three gestures of the right upper limb in terms of ME and MI [15]. The group considered the EEG patterns produced during each individual gesture, rather than the binary approach taken in this project. They constructed an adaptive CSP to filter 3-class calibration data by using the One-Versus-All (OVA) approach. They reported an online accuracy of $64.8 \%$ for their 3-class MI problem with similarly poorer results for their ME comparison, $61.9 \%$. The performance of the hybrid BCI designed in this project saw a $12.5 \%$ and $12.9 \%$ increase in MI and ME online test session accuracy, respectively. Most likely owing to the two-class problem being easier to solve.

It is not known the exact accuracy at which a BCI will induce neuroplasticity. However, as Jochumsen et al. showed with their two-class MI approach, a system performance of around $60-70 \%$ is enough to result in an increase in cortical projections of target muscle when combined with simultaneous functional electrical stimulation [25]. The average classification of the MI-SSVEP hybrid BCI designed in this project was $77.2 \%$, implying that the system could be beneficial in a neurorehabilitation setting. Despite the average accuracy, however, there were some participants who performed at the lower end of the accuracy range offered by Jochumsen $e t$ al. This illustrates the need for patient screenings, as not every person may benefit from a hybrid BCI system, reflecting the phenomenon known as 'BCI illiteracy'. However, as a concept, BCI illiteracy may be problematic. Thompson pointed out in [17] that it perhaps wrongly implies that some subjects are inherently incapable of BCI proficiency, removing the onus from engineers to build more inclusive BCIs.

Nevertheless, the project has demonstrated the feasibility of a MI-SSVEP hybrid in stroke rehabilitation. Short calibration sessions can both train the classifiers and allow the patients to become familiar with the training tasks. The system could be customized on the advice of a therapist to provide their patients with a more individualised therapy by increasing or reducing the number of gestures or by changing the gesture type.

From the outset of this project, the hybrid training paradigm was envisioned as a supplement to a FES rehabilitation strat- 
egy, as rich afferent feedback is an essential part of promoting neuroplasticity [13]. Presently, the standard form of FES rehabilitation strategies is mostly a passive activity with only a tenuous link between paretic limb stimulation and mental activities [26]. The system devised in this project, however, could infer a user's intention to trigger a specific stimulation pattern and output this intention to an FES device, synchronising functional motion with modulated brain activity. Furthermore, this project was mostly concerned with the accuracies possible with a hybrid system and not with making actual decisions, or providing an external device with a command. However, as Figure 3 shows, the accuracy is stable throughout a trial, implying that this type of classification could be used to make a decision in an asynchronous system by implementing a dwell time. Müller-Putz et al. employed a dwell-time system in their SSVEP-based BCI to allow for the control of an electrical prosthesis [27]. They achieved this by requiring a correct classification to be made for at least 1 second, or 4 iterations of a sliding window, triggering a refractory period where the system would make no further decisions, and the control signal was sent to the electrical prosthetic. This method allowed for asynchronous control, which offers a more pleasant user experience over the synchronous fixed-trial method as it allows the user to complete the training in their own time. Based on the method used in [27], the system designed in this project could be fashioned in a similar way. For example, if a cue is presented for a palmar grasp, and the system classifies the grasp correctly for multiple consecutive windows, the system could then send a stimulation pattern to an FES device. The BCI devised in this project used 3 classes for its SSVEP condition, relating to 3 single-limb gestures, but it could easily be extended to many more by adding additional LEDs at different flickering frequencies.

Ideally, there would be no need for the inclusion of a secondary mental task. Ideally, patients could trigger a multitude of control signals based on MI effort alone. Given the poor spatial resolution of EEG, perhaps the future should see a shift in focus towards other neuroimaging modalities more suited to the task. In 2018, using MEG, Barratt et al. showed that oscillatory beta responses to moving an index and pinky finger of the same hand were spatially separable for participants wearing a head-cast [28]. There are various clinical obstacles to overcome when using MEG but this level of separability is enviable amongst EEG researchers.

A general factor to consider when drawing conclusions from our results, and indeed results from the majority of MI-BCI studies, is that most, if not all of the participants in these experiment are using BCI for the first time. The ability to modulate SMR is a skill not immediately possessed by everyone (BCI illiteracy/inefficiency). Indeed, as a skill, it can be improved through practice [29]. Therefore, accuracies reported in this article and indeed many others of a similar nature should not be taken as a practical limit, this would be to ignore the learning curve that comes with SMR modulation and may be akin to judging a person's cycling abilities after their very first go on a bike.

\section{Conclusions}

In this pilot study, we have successfully implemented a MI and SSVEP based hybrid BCI system that could potentially be used for upper limb neurorehabilitation of stroke patients. The system performance achieved a classification accuracy of $77.3 \pm 8.2 \%$ (hybrid), with a SSVEP classification accuracy of $94.4 \pm 3.5 \%$, and MI classification accuracy of $80.9 \pm 8.1 \%$, comparable to the accuracies of the purely MI-based 2-class BCIs reported in literature.

Overall, the feasibility of the hybrid BCI paradigm was confirmed, with results suggesting the system could be used in a neurorehabilitation environment, with little to no additional cost to the user over purely MI BCIs. Despite the encouraging results there is still much work to be done before such a system is embraced by the clinical world. Work needs to be done to devise an engaging and natural-feeling training strategy that will motivate patients to actively participate. In addition, practicalities such as channel reduction and selection must be addressed and considerations have to be made in regards to calibration time if BCI paradigms are to make it out of the laboratory and into the clinic.

\section{ACKNOWLEDGEMENTS}

The authors would like to thank the Hearing Systems Group at the Department of Electrical Engineering, DTU, for providing us the EEG facility for data collection. Special thanks to Bastian Epp, for continued support to use their EEG facility. We acknowledge the PhD student, Jakob Møller, for his support throughout the project. Lastly, thanks go to the subjects who volunteered to participate in this study.

\section{REFERENCES}

[1] V. L. Feigin, M. H. Forouzanfar, R. Krishnamurthi, G. A. Mensah, M. Connor, D. A. Bennett, A. E. Moran, R. L. Sacco, L. Anderson, T. Truelsen et al., "Global and regional burden of stroke during 19902010: findings from the global burden of disease study 2010," The Lancet, vol. 383, no. 9913, pp. 245-255, 2014.

[2] S. Prabhakaran, I. Ruff, and R. A. Bernstein, "Acute stroke intervention: a systematic review," JAMA, vol. 313, no. 14, pp. 1451-1462, 2015.

[3] S. C. Cramer, M. Sur, B. H. Dobkin, C. O’brien, T. D. Sanger, J. Q Trojanowski, J. M. Rumsey, R. Hicks, J. Cameron, D. Chen et al., "Harnessing neuroplasticity for clinical applications," Brain, vol. 134, no. 6, pp. 1591-1609, 2011.

[4] S. De Vries and T. Mulder, "Motor imagery and stroke rehabilitation: a critical discussion," J. Rehabil. Med., vol. 39, no. 1, pp. 5-13, 2007.

[5] B. Graimann and G. Pfurtscheller, "Quantification and visualization of event-related changes in oscillatory brain activity in the time-frequency domain," Prog. brain res., vol. 159, pp. 79-97, 2006.

[6] M. Cirstea and M. Levin, "Improvement of arm movement patterns and endpoint control depends on type of feedback during practice in stroke survivors," Neurorehabil. Neural Repair, vol. 21, no. 5, pp. 398-411, 2007.

[7] F. A. Jure, L. C. Carrere, G. G. Gentiletti, and C. B. Tabernig, "Bci-fes system for neuro-rehabilitation of stroke patients," in J. Phys. Conf. Ser., vol. 705, no. 1. IOP Publishing, 2016, p. 012058.

[8] S. R. Soekadar, N. Birbaumer, and L. G. Cohen, "Brain-computer interfaces in the rehabilitation of stroke and neurotrauma," in Systems Neuroscience and Rehabilitation. Springer, 2011, pp. 3-18.

[9] S. Jiang, L. Chen, Z. Wang, J. Xu, C. Qi, H. Qi, F. He, and D. Ming, "Application of bci-fes system on stroke rehabilitation," in Neural Engineering (NER), 2015 7th International IEEE/EMBS Conference on. IEEE, 2015, pp. 1112-1115. 
[10] A. Vuckovic, M. Fraser, L. Wallace, and B. C. Osuagwu, "Braincomputer interface and functional electrical stimulation for neurorehabilitation of hand in sub-acute tetraplegic patients-functional and neurological outcomes," 2015.

[11] Y. Y. Jang, T. H. Kim, and B. H. Lee, "Effects of brain-computer interface-controlled functional electrical stimulation training on shoulder subluxation for patients with stroke: A randomized controlled trial,' Occup. Ther. Int., vol. 23, no. 2, pp. 175-185, 2016.

[12] A. Ramos-Murguialday, D. Broetz, M. Rea, L. Läer, Ö. Yilmaz, F. L. Brasil, G. Liberati, M. R. Curado, E. Garcia-Cossio, A. Vyziotis et al., "Brain-machine interface in chronic stroke rehabilitation: a controlled study," Ann. Neurol., vol. 74, no. 1, pp. 100-108, 2013.

[13] A. Biasiucci, R. Leeb, I. Iturrate, S. Perdikis, A. Al-Khodairy, T. Corbet, A. Schnider, T. Schmidlin, H. Zhang, M. Bassolino et al., "Brainactuated functional electrical stimulation elicits lasting arm motor recovery after stroke," Nat. Commun., vol. 9, no. 1, p. 2421, 2018.

[14] B. J. Edelman, B. Baxter, and B. He, "Eeg source imaging enhances the decoding of complex right-hand motor imagery tasks," IEEE Trans. Biomed. Eng., vol. 63, no. 1, pp. 4-14, 2016.

[15] A. Palma, J. Moller, S. Puthusserypady, and H. K. Iversen, "An adaptive CSP filter to investigate user independence in a 3-class MI-BCI paradigm," Comput. Biol. Med. (In Press), 2018.

[16] L. M. McCane, E. W. Sellers, D. J. McFarland, J. N. Mak, C. S. Carmack, D. Zeitlin, J. R. Wolpaw, and T. M. Vaughan, "Brain-computer interface (bci) evaluation in people with amyotrophic lateral sclerosis," Amyotroph. Lateral Scler. Frontotemporal Degener, vol. 15, no. 3-4, pp. 207-215, 2014.

[17] M. C. Thompson, "Critiquing the concept of bci illiteracy," Sci. Eng. Ethics, pp. 1-17, 2018.

[18] G. Pfurtscheller, B. Z. Allison, C. Brunner, G. Bauernfeind, T. SolisEscalante, R. Scherer, T. O. Zander, G. Mueller-Putz, C. Neuper, and N. Birbaumer, "The hybrid bci," Front. Neurosci., vol. 4, 2010.

[19] A. M. Savić, N. M. Malešević, and M. B. Popović, "Feasibility of a hybrid brain-computer interface for advanced functional electrical therapy," ScientificWorldJournal., vol. 2014, 2014.

[20] C. Brunner, B. Allison, C. Altstätter, and C. Neuper, "A comparison of three brain-computer interfaces based on event-related desynchronization, steady state visual evoked potentials, or a hybrid approach using both signals," J. Neural Eng., vol. 8, no. 2, p. 025010, 2011.

[21] L. F. Nicolas-Alonso and J. Gomez-Gil, "Brain computer interfaces, a review," Sensors, vol. 12, no. 2, pp. 1211-1279, 2012.

[22] H. Gollee, I. Volosyak, A. J. McLachlan, K. J. Hunt, and A. Gräser, "An ssvep-based brain-computer interface for the control of functional electrical stimulation," IEEE Trans. Biomed. Eng., vol. 57, no. 8, pp. $1847-1855,2010$

[23] H. Ramoser, J. Muller-Gerking, and G. Pfurtscheller, "Optimal spatial filtering of single trial eeg during imagined hand movement," IEEE Trans. Biomed. Eng., vol. 8, no. 4, pp. 441-446, 2000.

[24] F. Lotte and C. Guan, "Regularizing common spatial patterns to improve bci designs: unified theory and new algorithms," IEEE Trans. Biomed. Eng., vol. 58, no. 2, pp. 355-362, 2011.

[25] M. Jochumsen, I. K. Niazi, D. Taylor, D. Farina, and K. Dremstrup, "Detecting and classifying movement-related cortical potentials associated with hand movements in healthy subjects and stroke patients from singleelectrode, single-trial eeg," J. Neural Eng., vol. 12, no. 5, p. 056013, 2015.

[26] B. M. Young, J. Williams, and V. Prabhakaran, "Bci-fes: could a new rehabilitation device hold fresh promise for stroke patients?" Expert Rev. Med. Devices, vol. 11, no. 6, pp. 537-539, 2014.

[27] G. R. Muller-Putz and G. Pfurtscheller, "Control of an electrical prosthesis with an ssvep-based bci," IEEE Trans. Biomed. Eng., vol. 55, no. 1, pp. 361-364, 2008.

[28] E. L. Barratt, S. T. Francis, P. G. Morris, and M. J. Brookes, "Mapping the topological organisation of beta oscillations in motor cortex using meg," NeuroImage, 2018.

[29] C. Jeunet, E. Jahanpour, and F. Lotte, "Why standard brain-computer interface (bci) training protocols should be changed: an experimental study," J. Neural Eng., vol. 13, no. 3, p. 036024, 2016. 